\title{
Caps on Political Lobbying: Comment
}

\author{
By Todd R. Kaplan AND David Wettstein ${ }^{1}$
}

Following the 1999 Israeli elections, the winning candidate's (Ehud Barak's) party was fined 3.2 million dollars for violating Israel's campaign financing laws by exceeding the spending cap imposed and raising 1.2 million dollars illegally (see Time Europe, 2000). While adhering to the strict NCAA recruiting regulations, the University of Oregon spent 3 million dollars to make their football locker room the "best anywhere, including the NFL" (Lexington Herald Leader, 2003). When asked about the previous salary cap in the UK Football league, players said that "it [the cap] was so artificial that clubs paid money in brown paper envelopes." (BBC2, Newsnight 2002).

In all these cases, there officially existed a rigid spending cap. However, they show that even though a cap exists: The cap might not be easily enforced. When enforced, the penalty may just be financial rather than a change in outcome. The cap may be enforced and adhered to, but there may also be alternate perhaps less effective (hence more costly) means of spending. We also see that the flexible nature of the cap is often built into the rules by examining the salary caps of the four major US sports leagues where the caps are routinely exceeded. In baseball and basketball, surpassing the cap invokes a pure financial penalty, called a luxury tax, which is proportional to the excess. In football, while the cap is stated in terms of salaries, many teams use signing bonuses as a way to circumvent it. In ice hockey, there is a dual cap system, where the luxury tax is invoked on salaries falling between the two caps and signing bonuses are an allowed method to exceed the 
upper cap.

Previous literature analyzed the imposition of caps in contests reaching the conclusion that caps potentially can paradoxically lead to increased spending. Yeon-Koo Che and Ian L. Gale (1998) analyze a complete information asymmetric environment and show that even with linear costs the paradox may occur. Arieh Gavious, Benny Moldovanu and Aner Sela (2003) study a symmetric, incomplete information environment and find for the case of convex costs, the paradox occurs.

In both of these papers in contrast to the above examples, the caps were rigid (fully enforced). Instead, we analyze environments where caps can be exceeded but at a higher cost (either punishments or other less efficient means of spending). Todd R. Kaplan, Israel Luski, Aner Sela and David Wettstein (2002) and Todd R. Kaplan, Israel Luski and David Wettstein (2003) study the effects of an increase in the cost function in an incomplete and complete information all-pay auction, respectively, and find that such an increase always leads to a decrease in bids.

In this comment, we examine the original Che and Gale model for the case of nonrigid caps and show the existence of a cap will indeed serve to reduce spending. We then proceed to discuss the welfare effects and policy implications of caps. We start by introducing the model in the next section. 


\section{The Model}

As with Che and Gale (1998, henceforth CG), we assume that there are two bidders: bidder 1 with value $v_{1}$ and bidder 2 with value $v_{2}$ where $v_{1} \geq v_{2}$. The cost of bidding $x$ includes the money and effort needed to generate the bid as well as the cost associated with the risk of punishment. We denote this expenditure (cost) as $c(x)$ and assume it is the same for each bidder, strictly increasing, continuous with $c(0)=0$. Note that this includes the linear case analyzed by CG where $c(x)=x$. The highest bidder wins the prize while all the bidders pay their bids (an all-pay auction). Similar to CG, we introduce a cap on (monetary) bidding. Unlike CG, we assume that it is possible to bid above the cap, but at some additional cost (either increased risk of punishment or greater effort). The effective cost of bidding with a cap is denoted by $\bar{c}(x)$ where $\bar{c}(x) \geq c(x)$ and the assumptions on $\bar{c}(x)$ are the same as the assumptions on $c(x)$.

Plausible explanations for why a bidder can potentially bid beyond a cap are as follows. One possibility is that the punishment mechanism is not foolproof and monetary expenditures are not perfectly observable, so (small) expenditures above the cap may go unpunished. Furthermore, the penalty for exceeding the cap may be a fine that is a continuous function of the excess monetary expenditure.

Finally, the bidding could be multi-dimensional. In addition to the monetary aspect, there could also be a non-pecuniary aspect such as effort. Money $m$ and effort $e$ spent are aggregated into a single score $w(m, e)$, where the winner is the individual with the highest

score. The imposition of a cap increases the effective cost of generating the score. ${ }^{1}$ The 
placement of the cap on money could shift expense from money into effort distorting the optimal composition of the bid.

We wish to emphasize that with one-dimensional bidding, $x$ represents monetary expenditure; with multi-dimensions, it represents the score that is a combination of monetary spending and effort. We now proceed to analyze the equilibrium behavior.

\section{Equilibrium Bidding}

The equilibrium behavior entails mixed-strategies over the same support that can be described by cumulative distributions $F_{1}$ and $F_{2}$ for bidders 1 and 2 , respectively. ${ }^{2}$ The following theorem fully characterizes equilibrium bidding and outcomes.

Theorem 1 Given any strictly increasing and continuous cost function $c$, with $c(0)=0$, the equilibrium mixed-strategies of the two bidders are given by the following cumulative distribution functions: $F_{1}(x)=\frac{c(x)}{v_{2}} ; \quad F_{2}(x)=\frac{v_{1}-v_{2}+c(x)}{v_{1}}$.

Proof. Each bidder must be indifferent over the support of his strategy. The equilibrium payoff for bidder 1 cannot fall below $v_{1}-v_{2}$, since he can bid an $x$ such that $c(x)=v_{2}$ and win with certainty. The bidders cannot both have atoms at the same point since an infinitesimal increase in bid will cause a discrete jump in the expected reward which leads to a discrete jump in the expected payoff due to the continuity of the cost function. This implies at least one bidder that bids the lowest possible equilibrium bid will have zero equilibrium payoff (there would be a zero probability of winning for a bidder not placing 
an atom). Since bidder 1 has positive payoff, this can only be bidder 2 . Hence, bidder 2 has zero payoff. This then implies that bidder 1's payoff cannot exceed $v_{1}-v_{2}$ since it would imply bidder 1 is bidding below $v_{2}$ and then bidder 2 can profitably undercut him. Thus, bidder 1's expected payoff is $v_{1}-v_{2}$ and equals his probability of winning $F_{2}(x)$ times $v_{1}$ minus his cost as reflected by:

$$
F_{2}(x) v_{1}-c(x)=v_{1}-v_{2}
$$

Similarly, using bidder 2's expected payoff equation, we obtain

$$
F_{1}(x) v_{2}-c(x)=0
$$

Together this yields,

$$
\begin{aligned}
& F_{1}(x)=\frac{c(x)}{v_{2}} \\
& F_{2}(x)=\frac{v_{1}-v_{2}+c(x)}{v_{1}}
\end{aligned}
$$

The straightforward derivation of the equilibrium bidding functions and their "simple" behavior is the consequence of assuming a continuous cost function following the imposition of the bid. In the CG model, the infinite cost jump allows for the existence of an atom at the cap. This creates technical difficulties such as a more elaborate equilibrium behavior and the possibility of non-uniqueness.

Corollary 1 The expected individual expenditure (and hence the expected aggregate expenditure) as well as the probability of either bidder winning is invariant to changes in the cost function. 
Proof. The expected individual costs are $E[c(x)]=\int_{0}^{c^{-1}\left(v_{2}\right)} c(x) d F_{1}=\frac{v_{2}}{2}$ and $E[c(x)]=\int_{0}^{c^{-1}\left(v_{2}\right)} c(x) d F_{2}=\frac{v_{2}^{2}}{2 v_{1}}$ for bidders 1 and 2, respectively. The probability of bidder 1 winning is $\int F_{2} d F_{1}=1-\frac{v_{2}}{2 v_{1}}$.

Even though increasing the cost function does not change expected expenditures, it does change expected bids, as the following Corollary shows.

Corollary 2 The bidding without a cap first-order stochastically dominates bidding with a cap; hence, the imposition of a cap reduces the expected bids.

Proof. We denote the equilibrium cumulative distributions for the case with the caps by $\bar{F}_{1}$ and $\bar{F}_{2}$. We then obtain the following equilibrium distributions.

$$
\begin{aligned}
& \bar{F}_{1}(x)=\frac{\bar{c}(x)}{v_{2}} \\
& \bar{F}_{2}(x)=\frac{v_{1}-v_{2}+\bar{c}(x)}{v_{1}}
\end{aligned}
$$

Since $\bar{c}(x) \geq c(x)$, we must have $\bar{F}_{1}(x) \geq F_{1}(x)$ and $\bar{F}_{2}(x) \geq F_{2}(x)$. Hence, the imposition of a cap in our environment reduces the expected bids.

\section{Welfare Implications}

The imposition of the caps has different welfare implications for the following three groups: the participants (bidders), the recipients of the bids and other members of society. As Corollary 1 shows, the welfare of the participants remains unchanged.

The recipients of the monetary bids would be worse off. Even in the multidimensional case, the other less-efficient dimensions are less desirable to the recipient; otherwise they 
would be the more-efficient means without the cap (the NCAA football player would prefer a monetary payment rather than use of a new gym).

The welfare consequences for other members of society are determined solely by the reallocation of the costs of bidding. Unlike CG, the bidders' winning probabilities are unchanged (hence, it does not matter whether or not participants' values for the "prize" are aligned with other members of society). The shifting of costs affects welfare ambiguously. If all such expenditure is wasteful (as one may argue in political campaigns), then there is no change. If part of the expenditure is collected in a fine, then these fines may be used to finance more productive activities than the bidding activities. However, if the bidding activities are valued and punishment is non-pecuniary (such as banning a team from future competition), then caps shifting expenditure into punishment will be detrimental. Also note, in the case of multi-dimensional bids, the less efficient bidding activity may be more socially desirable. For other members of society it might be better to bribe student athletes with new gyms rather than with money.

\section{Conclusion}

Rigid bid caps are not always fully enforced in models of political competition, sports and professional recruiting. Instead, mitigating the effects of the cap is possible but costly. We find that under such an assumption, contrary to previous literature, the imposition of a cap will always decrease monetary spending while total expenditure stays the same. 


\section{References}

[1] BBC2 Newsnight, "Interview with Alex Finn (consultant) and Gordon Taylor (players' representative)," 19 March 2002.

[2] Che, Yeon-Koo and Gale, Ian L. "Caps on Political Lobbying." American Economic Review, June 1988, 88(3), pp. 643-51.

[3] Gavious, Arieh; Moldovanu, Benny and Sela, Aner. "Bid Costs and Endogenous Bid Caps." Rand Journal of Economics, Winter 2002, 33(4), pp. 709-22.

[4] Kaplan, R. Todd; Luski, Israel; Sela, Aner and Wettstein, David. "All-Pay Auctions with Variable Rewards." Journal of Industrial Economics, December 2002, 50(4), pp. $417-30$

[5] Kaplan, R. Todd; Luski, Israel and Wettstein, David. "Innovative Activity and Sunk Cost," International Journal of Industrial Organization, October 2003, 21(8), pp. $1111-33$.

[6] Lexington Herald Leader, "The University of Oregon's football locker room is a showcase of frivolous one-upmanship," 18 September 2003.

[7] Time Europe "A Blow to Barak's Image," February 7, 2000. Vol. 155, No. 5. www.time.com/time/europe/magazine/2000/27/barak.html 


\section{Notes}

1* Kaplan: Department of Economics, University of Exeter, Exeter, EX4 4PU, UK, email: Dr@ToddKaplan.com; Wettstein: Department of Economics Ben-Gurion University of the Negev, Monaster Center for Economic Research, Beer-Sheva 84105, Israel, email: wettstn@bgu.ac.il. . We are grateful to two anonymous referees for several comments and suggestions.

${ }^{1}$ Denote $c(m, e)$ as the cost of spending $m$ with effort $e$. The bidders want to minimize this cost for achieving a given bid (score) $x$. Their effective cost $c(x)$ is then

$$
c(x)=\min _{m, e} c(m, e) \text { s.t. } w(m, e)=x
$$

One can impose caps on money, but not on effort or score. The effective cost $\bar{c}(x)$ under a monetary cap $\bar{m}$ is then

$$
\bar{c}(x)=\min _{m, e} c(m, e) \text { s.t. } w(m, e)=x \text { and } m \leq \bar{m}
$$

We thus have $\bar{c}(x) \geq c(x)$.

${ }^{2}$ See Kaplan et al. (2003), Appendix A, for a detailed proof that a pure-strategy equilibrium cannot exist. 